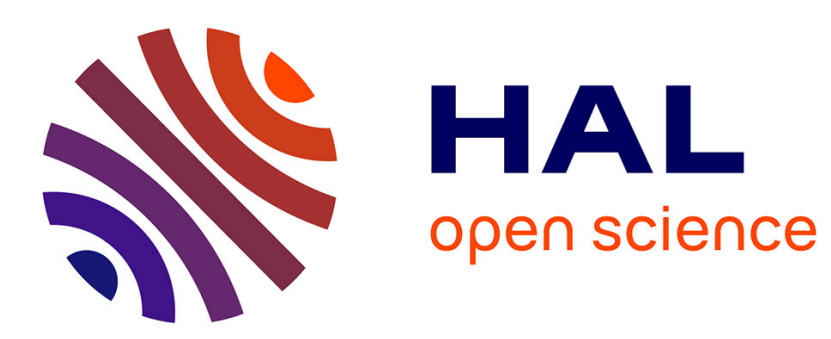

\title{
The decay of stabilizability with Reynolds number in a linear model of spatially developing flows
}

\author{
Eric Lauga, Thomas Bewley
}

\section{To cite this version:}

Eric Lauga, Thomas Bewley. The decay of stabilizability with Reynolds number in a linear model of spatially developing flows. Proceedings of the Royal Society A: Mathematical, Physical and Engineering Sciences, 2003, 459 (2036), pp.2077-2095. 10.1098/rspa.2002.1116 . hal-01024924

HAL Id: hal-01024924

https://hal-polytechnique.archives-ouvertes.fr/hal-01024924

Submitted on 8 Dec 2017

HAL is a multi-disciplinary open access archive for the deposit and dissemination of scientific research documents, whether they are published or not. The documents may come from teaching and research institutions in France or abroad, or from public or private research centers.
L'archive ouverte pluridisciplinaire HAL, est destinée au dépôt et à la diffusion de documents scientifiques de niveau recherche, publiés ou non, émanant des établissements d'enseignement et de recherche français ou étrangers, des laboratoires publics ou privés. 


\title{
The decay of stabilizability with Reynolds number in a linear model of spatially developing flows
}

\author{
By ERIC LAUGA ${ }^{1,2} \dagger$ And Thomas R. BeWLeY \\ ${ }^{1}$ Flow Control Laboratory, Department of Mechanical and Aerospace Engineering, \\ University of California San Diego, La Jolla, CA 92093, USA \\ ${ }^{2}$ LadHyX, École Polytechnique-CNRS, 91128 Palaiseau Cedex, France
}

This article characterizes the gradual decay of stabilizability with Reynolds number in the linear complex Ginzburg-Landau model of spatially developing flow systems when a single spatially-localized actuator is used to apply the control forcing. It is shown that, technically, the system considered is linearly stabilizable for all actuator locations at any Reynolds number. However, as the Reynolds number is increased and an increasing number of modes of the open-loop system become unstable, the control authority on some of these open-loop unstable modes is found to be exponentially small. Using finite-precision arithmetic and any given numerical method for computing the feedback gains, an effective upper bound on the Reynolds number is reached, above which it is not possible to compute a linearly stabilizing control algorithm. This 'effective upper bound', however, is not a fundamental characteristic of the system; rather, it is a persistent artefact of the numerical precision used in the controller calculation. The most suitable location for the actuator as the Reynolds number is increased is well predicted by analysis of the domain of support of the open-loop adjoint eigenfunctions. Further understanding is provided by analysis of the closed-loop system eigenfunctions, which are shown to become increasingly nonnormal as the Reynolds number is increased.

Keywords: flow control; instability of shear flows; loss of stabilizability

\section{Introduction}

Spatially developing open shear-flow instabilities have been extensively studied in the last fifteen years using the concepts of local and global instability (Huerre \& Monkewitz 1990). In the laboratory reference frame (chosen without ambiguity because of inlet conditions or the presence of a body in the flow), the existence of a mean advection velocity allows the local flow instabilities to be either of convective type, when the advection is strong enough to wash downstream all growing perturbations, or of absolute type, when the local flow instabilities grow in both the upstream and downstream directions.

$\dagger$ Present address: Division of Engineering and Applied Sciences, Harvard University, Pierce Hall, 29 Oxford St, Cambridge, MA 02138, USA. 
Flows displaying a sufficiently large region of local absolute instability will typically display self-sustained unsteadiness, as is the case with mixing layers (Huerre \& Monkewitz 1985), jets (Monkewitz \& Sohn 1988) and wakes (Monkewitz 1988). In such systems, when a physical bifurcation parameter (such as the Reynolds number) exceeds a critical value, an eigenfunction of the linearized governing equations, sometimes termed a linear global mode, is linearly unstable and thus small system disturbances inevitably trigger unsteady flow behaviour. Due to stabilizing nonlinear effects, this unsteadiness typically 'saturates' into a 'self-sustained' finite-amplitude limit cycle, sometimes termed a nonlinear global mode. In the well-known case of the cylinder wake, self-sustained oscillations appear downstream of the body when the Reynolds number based on the cylinder diameter exceeds 47 . In this system, the nonlinear global mode is referred to as the von Karman vortex street.

The important engineering consequences of delaying the appearance of selfsustained unsteady flow phenomena, and the recent successful control implementations in both experimental (Roussopoulos 1993) and numerical (Park et al. 1994; Min \& Choi 1999) investigations of such systems, has led to the following fundamental questions. Is there an effective upper bound on the bifurcation parameter, above which the system cannot be linearly stabilized with any control strategy for a given actuator configuration? If so, why? The present article is devoted to these two questions.

\section{The Ginzburg-Landau model of weakly non-parallel flows}

As a model of inhomogeneous weakly non-parallel flows, the complex GinzburgLandau (CGL) equation has proven to be particularly well suited in several previous studies (see, for example, Monkewitz et al. 1993; Pier \& Huerre 2001). In particular, the CGL model has proven to be accurate in determining global frequency criteria in both the linear (Chomaz et al. 1991) and nonlinear (Pier et al. 1998) regimes. Moreover, because the CGL model roughly captures the streamwise structure of the system eigenfunctions and the variation of the complex frequency of these eigenmodes with Reynolds number, the CGL model has also allowed quantitative predictions of the effects of proportional feedback control on the actual flow system in several previous studies (Monkewitz 1989, 1993; Monkewitz et al. 1991; Roussopoulos \& Monkewitz 1996). In this paper, we thus consider the CGL model of the flow exclusively. By so doing, we may focus our attention directly on the fundamental issues responsible for the decay of stabilizability with $R e$ in such systems.

The linear CGL equation considered in this work models the time evolution of a perturbation quantity $\psi$ in the presence of streamwise advection, amplification, diffusion and control input,

$$
\frac{\partial \psi}{\partial t}+U \frac{\partial \psi}{\partial x}=\mu \psi+\nu \frac{\partial^{2} \psi}{\partial x^{2}}+\delta\left(x-x_{\mathrm{f}}\right) u \quad \Leftrightarrow \quad \frac{\partial \psi}{\partial t}=\mathcal{L} \psi+\delta\left(x-x_{\mathrm{f}}\right) u .
$$

In order to model a wide range of spatially developing flow systems, the coefficients $\mu=\mu(\epsilon x)$ and $\nu$ in this equation are allowed to be complex. Note that the scalar control input $u=u(t)$ is modelled as a pointwise forcing term at $x=x_{\mathrm{f}}$. The amplification term in this model equation depends on an inhomogeneous local parameter $\mu(\epsilon x)$ analogous to the local Reynolds number based on, for example, the local diameter and velocity defect of a three-dimensional bluff-body wake. In the present work, 
the spatial variation of this coefficient is assigned the form $\mu(\epsilon x)=\mu_{0}-\left[\epsilon\left(x-x_{t}\right)\right]^{2}$, where the real parameter $\mu_{0}$ is a global bifurcation parameter analogous to the global Reynolds number based on, for example, the bluff-body dimension and free-stream velocity, and the small parameter $\epsilon$ accounts for the slowly diverging characteristics of the basic flow. The spatial position $x_{t}$, which is generally taken to be complex, is found by analytic continuation of local dispersion relations (Hammond \& Redekopp 1997), and characterizes the hydrodynamic resonance phenomenon (Chomaz et al. 1991). The parabolic form used here is motivated by many previous studies, which focused on the modelling of spatially developing flows (Chomaz et al. 1987, 1990; Huerre \& Monkewitz 1990; Roussopoulos \& Monkewitz 1996). Using this parabolic form, it was shown by Chomaz et al. (1987) that local instability appears in a finite region in the system when $\mu_{0}>0$, this local instability being everywhere convective if $\mu_{0}<\mu_{\mathrm{a}} \triangleq U^{2} \operatorname{Re}(\nu) / 4|\nu|^{2}$, and absolute in a portion of the unstable region if $\mu_{0}>\mu_{\mathrm{a}}$. Significantly, the localized control forcing term applied to (2.1) does not change these local instability properties of the system, though it can substantially alter its global dynamics.

The analytic solution (Chomaz et al. 1987) for the eigenmodes $\psi(x, t)=\xi(x) \mathrm{e}^{\lambda t}$ of the unforced CGL equation is given by the countable set of eigenvalues $\lambda_{k}$ and corresponding eigenfunctions $\xi_{k}(x)$,

$$
\left.\begin{array}{rl}
\lambda_{k} & =\mu_{0}-\frac{U^{2}}{4 \nu}-\epsilon \nu^{1 / 2}(2 k+1), \\
\xi_{k}(x) & =\exp \left[\frac{U x}{2 \nu}-\frac{\epsilon\left(x-x_{t}\right)^{2}}{2 \nu^{1 / 2}}\right] \operatorname{He}_{k}\left[\left(\frac{4 \epsilon^{2}}{\nu}\right)^{1 / 4}\left(x-x_{t}\right)\right],
\end{array}\right\} \quad k=0,1,2, \ldots,
$$

where $\mathrm{He}_{k}$ designs the $k$ th Hermite polynomial. The first mode is linearly unstable (i.e. $\operatorname{Re}\left(\lambda_{0}\right)>0$ ) when the supercriticality $\delta \triangleq\left(\mu_{0}-\mu_{\mathrm{c}}\right) / \mu_{\mathrm{c}}$ is positive, with $\mu_{\mathrm{c}} \triangleq \mu_{\mathrm{a}}+\epsilon \operatorname{Re}\left(\nu^{1 / 2}\right)$. The fact that $\mu_{\mathrm{c}}>\mu_{\mathrm{a}}$ confirms the need for a sufficiently large region of local absolute instability to give rise to global instability (Chomaz et al. 1991). Note that the shapes of these modes do not depend on the global bifurcation parameter $\mu_{0}$.

The numerical values used in this paper are $U=6, \nu=1-10 \mathrm{i}$ and $x_{t}=0.1 \mathrm{i}$, to be consistent with previous studies (Chomaz et al. 1987, 1990). The value $\epsilon=0.01$ was chosen to represent a weakly inhomogeneous media, corresponding to a ratio of a typical local instability wavelength $\lambda$ to a global length-scale $L$ of $\lambda / L \approx 0.1$. This choice is physically realistic; for example, inside the wake behind a rectangular body, this ratio typically varies between 0.05 and 0.2 (Hammond \& Redekopp 1997).

Designating by $\boldsymbol{x}, A$ and $B$ the spatial discretizations of the state variable $\psi$, the Ginzburg-Landau operator $\mathcal{L}$ and the Dirac delta function $\delta\left(x-x_{\mathrm{f}}\right)$, respectively, it is straightforward to represent a discretization of the CGL equation (2.1) in the standard state-space form,

$$
\dot{\boldsymbol{x}}=A \boldsymbol{x}+B \boldsymbol{u} .
$$

In the present work, an efficient spatial discretization of the CGL equation was achieved using a Fourier collocation method on a stretched grid clustered near the forcing location. Other numerical schemes were also implemented for comparison and achieved the same results upon grid refinement. 


\section{Stabilizability}

The CGL system (2.1) is termed 'stabilizable' if and only if a control strategy coordinating $u$ with $\psi$ can be found such that the controlled linear system eventually decays exponentially in time everywhere in space regardless of initial conditions. In the discrete setting, the system operator pair $(A, B)$ in $(2.3)$ is termed stabilizable if and only if a feedback rule $\boldsymbol{u}=K \boldsymbol{x}$ may be found such that all of the eigenvalues of $A+B K$ have negative real part. In the present section, we discuss three equivalent tests for determining whether or not the CGL system (2.1), or its discretization (2.3), is stabilizable.

(i) Checking (analytically) whether or not the 'controllability matrix' corresponding to the dynamic equation for the unstable modes of the system (2.1) has full rank.

(ii) Checking (numerically) whether or not the discretized optimal control problem given by minimization of $\mathcal{J}=\int_{0}^{\infty}\left(\boldsymbol{x}^{*} Q \boldsymbol{x}+\boldsymbol{u}^{*} R \boldsymbol{u}\right) \mathrm{d} t$ with feedback of the form $\boldsymbol{u}=K \boldsymbol{x}$ has a stabilizing solution, where $Q>0$ and $R>0$ and $\boldsymbol{x}$ and $\boldsymbol{u}$ are related by $(2.3)$.

(iii) Checking (numerically) whether or not a minimal-energy stabilizing control feedback rule may be found by pole placement, resulting in a closed-loop system matrix that reflects the unstable eigenvalues of $A$ to the left half-plane and leaves the stable eigenvalues of $A$ unchanged.

\section{(a) Analytic determination of the controllability of the unstable modes}

At any time $t$, the solution $\psi(x, t)$ of the CGL equation is spanned by the eigenfunctions $\xi_{\kappa}(x)$, also known as 'linear global modes' in much of the CGL literature. Recall that the $\xi_{\kappa}$ are available analytically, and the eigenvalues $\lambda_{\kappa}$ are distinct. We now define the 'adjoint eigenfunctions' $\eta_{\kappa}(x)$ (also known as 'adjoint global modes' in much of the CGL literature) by changing $\{U, \mu, \nu\} \rightarrow\left\{-U, \mu^{*}, \nu^{*}\right\}$ in the unforced CGL equation, resulting in

$$
\frac{\partial \tilde{\psi}}{\partial t}-U \frac{\partial \tilde{\psi}}{\partial x}=\mu^{*} \tilde{\psi}+\nu^{*} \frac{\partial^{2} \tilde{\psi}}{\partial x^{2}} \Leftrightarrow \frac{\partial \tilde{\psi}}{\partial t}=\mathcal{L}^{*} \tilde{\psi}
$$

and solving for the eigenmodes analytically, resulting in the complex conjugate of the eigenvalues found previously, $\lambda_{\kappa}^{*}$, and the adjoint eigenfunctions

$$
\eta_{\kappa}(x)=\exp \left[-\frac{U x}{2 \nu^{*}}-\frac{\epsilon\left(x-x_{t}^{*}\right)^{2}}{2\left(\nu^{*}\right)^{1 / 2}}\right] \mathrm{He}_{\kappa}\left[\left(\frac{4 \epsilon^{2}}{\nu^{*}}\right)^{1 / 4}\left(x-x_{t}^{*}\right)\right], \quad \kappa=0,1,2, \ldots
$$

Defining the inner product $\left\langle\eta_{\iota}, \xi_{\kappa}\right\rangle=\int_{-\infty}^{\infty} \eta_{\iota}^{*}(x) \xi_{\kappa}(x) \mathrm{d} x$, note that

$$
\left.\begin{array}{l}
\left\langle\eta_{\iota}, \mathcal{L} \xi_{\kappa}\right\rangle=\left\langle\eta_{\iota}, \lambda_{\kappa} \xi_{\kappa}\right\rangle=\lambda_{\kappa}\left\langle\eta_{\iota}, \xi_{\kappa}\right\rangle, \\
\left\langle\eta_{\iota}, \mathcal{L} \xi_{\kappa}\right\rangle=\left\langle\mathcal{L}^{*} \eta_{\iota}, \xi_{\kappa}\right\rangle=\left\langle\lambda_{\iota}^{*} \eta_{\iota}, \xi_{\kappa}\right\rangle=\lambda_{\iota}\left\langle\eta_{\iota}, \xi_{\kappa}\right\rangle,
\end{array}\right\} \quad\left(\lambda_{\kappa}-\lambda_{\iota}\right)\left\langle\eta_{\iota}, \xi_{\kappa}\right\rangle=0,
$$

and thus $\left\langle\eta_{\iota}, \xi_{\kappa}\right\rangle=0$ for $\iota \neq \kappa$. Scaling the eigenfunctions appropriately, it follows that $\left\langle\eta_{\iota}, \xi_{\kappa}\right\rangle=\delta_{\iota \kappa}$. 
In order to establish a correspondence between the analytically determined eigenfunctions and adjoint eigenfunctions discussed above and the appropriately defined discrete right and left eigenvectors, it is necessary to define a discrete scalar product $\tilde{\boldsymbol{x}}^{*} \mathcal{Q} \boldsymbol{x}$, which is equivalent to its continuous analogue, the inner product $\left\langle\eta_{\iota}, \xi_{\kappa}\right\rangle=\int_{-\infty}^{\infty} \eta_{\iota}^{*}(x) \xi_{\kappa}(x) \mathrm{d} x$. Using the definition of the (stretched) numerical grid and a trapezoidal integration rule, it is straightforward to compute the diagonal matrix $\mathcal{Q}>0$ that accomplishes this task. The discrete analogue of the eigenfunction $\xi_{\kappa}(x)$ is then found to be the $\kappa$ th right eigenvector of $A$, which we will denote $\boldsymbol{r}^{(\kappa)}$, and the discrete analogue of the adjoint eigenfunction $\eta_{\kappa}(x)$ is the $\kappa$ th left eigenvector of $\mathcal{Q} A \mathcal{Q}^{-1}$, which we will denote $\boldsymbol{s}^{(\kappa)} . \dagger$ Using this definition, it follows that

$$
\left.\begin{array}{rl}
\left(\boldsymbol{s}^{(\iota)}\right)^{*} \mathcal{Q} A \boldsymbol{r}^{(\kappa)} & =\left(\boldsymbol{s}^{(\iota)}\right)^{*} \mathcal{Q} \lambda_{\kappa} \boldsymbol{r}^{(\kappa)} \\
& =\lambda_{\kappa}\left(\boldsymbol{s}^{(\iota)}\right)^{*} \mathcal{Q} \boldsymbol{r}^{(\kappa)}, \\
\left(\boldsymbol{s}^{(\iota)}\right)^{*} \mathcal{Q} A \boldsymbol{r}^{(\kappa)} & =\left[\left(\boldsymbol{s}^{(\iota)}\right)^{*} \mathcal{Q} A \mathcal{Q}^{-1}\right] \mathcal{Q} \boldsymbol{r}^{(\kappa)} \\
& =\lambda_{\iota}\left(\boldsymbol{s}^{(\iota)}\right)^{*} \mathcal{Q} \boldsymbol{r}^{(\kappa)},
\end{array}\right\} \quad\left(\lambda_{\kappa}-\lambda_{\iota}\right)\left(\boldsymbol{s}^{(\iota)}\right)^{*} \mathcal{Q} \boldsymbol{r}^{(\kappa)}=0,
$$

and thus the discrete analogue of the orthogonality property $\left\langle\eta_{\iota}, \xi_{\kappa}\right\rangle=\delta_{\iota \kappa}$ is the discrete relationship $\left(\boldsymbol{s}^{(\iota)}\right)^{*} \mathcal{Q} \boldsymbol{r}^{(\kappa)}=\delta_{\iota \kappa}$.

Consider now a particular value of $\mu_{0}$ and $x_{\mathrm{f}}$ for which the CGL system has $n$ unstable eigenmodes. Decompose $\psi$ such that

$$
\psi(x, t)=\sum_{k=1}^{n} \xi_{k}(x) \chi_{k}(t)+\phi(x, t), \quad \text { with } \phi \in \mathrm{N}\left(\operatorname{span}\left\{\xi_{1}, \xi_{2}, \ldots, \xi_{n}\right\}\right),
$$

i.e. $\phi$ lies in the nullspace of the space spanned by $\left\{\xi_{1}, \xi_{2}, \ldots, \xi_{n}\right\}$. Taking the inner product of the adjoint eigenfunction $\eta_{\kappa}(x)$ with the CGL equation and applying this decomposition and the orthogonality property derived above, we obtain

$$
\begin{aligned}
\left\langle\eta_{\kappa}(x),\left[\frac{\partial \psi}{\partial t}=\right.\right. & \left.\left.\mathcal{L} \psi+\delta\left(x-x_{\mathrm{f}}\right) u\right]\right\rangle \\
& \Rightarrow \frac{\mathrm{d} \chi_{\kappa}}{\mathrm{d} t}=\lambda_{\kappa} \chi_{\kappa}+\left\langle\eta_{\kappa}(x), \delta\left(x-x_{\mathrm{f}}\right)\right\rangle u=\lambda_{\kappa} \chi_{\kappa}+\eta_{\kappa}\left(x_{\mathrm{f}}\right) u .
\end{aligned}
$$

Assembling these dynamic equations for all of the unstable modal coefficients $\chi_{\kappa}$ in vector form, we obtain the diagonal system

$$
\frac{\mathrm{d} \chi}{\mathrm{d} t}=\Lambda \chi+\bar{B} u, \quad \Lambda=\left(\begin{array}{ccc}
\lambda_{1} & & 0 \\
& \ddots & \\
0 & & \lambda_{n}
\end{array}\right), \quad \bar{B}=\left(\begin{array}{c}
\eta_{1}\left(x_{\mathrm{f}}\right) \\
\vdots \\
\eta_{n}\left(x_{\mathrm{f}}\right)
\end{array}\right) .
$$

This is an analytic expression of the dynamic equation for the unstable modes of the system.

$\dagger$ Note that the eigenvalues of the resolved modes of both $A$ and $\mathcal{Q} A \mathcal{Q}^{-1}$ are accurate approximations of the analytically determined eigenvalues in the continuous case in (2.2). Note also that, as is also true in the continuous case, all discrete left and right eigenvectors are enumerated by the real part of their corresponding eigenvalues in the open-loop setting, with the least-stable eigenmodes listed first. 
We now review the following facts.

(i) A system is state stabilizable if and only if all unstable modes are state controllable.

(ii) $(\Lambda, \bar{B})$ is state controllable if and only if the matrix

$$
C=\left(\begin{array}{lllll}
\bar{B} & \Lambda \bar{B} & \Lambda^{2} \bar{B} & \cdots & \Lambda^{n-1} \bar{B}
\end{array}\right)
$$

has full row rank.

In the present case, as $\Lambda$ is diagonal and $\lambda_{i} \neq 0 \forall i$, it follows that $C$ has full row rank if and only if the vector $\bar{B}$ has all non-zero entries. The adjoint eigenfunctions of the CGL equation, with the coefficients selected as described in $\S 2$, are non-zero everywhere on the real axis (their zeros lie in the complex plane). Thus, technically, the CGL system considered in this paper is stabilizable for any value of $\mu_{0}$ and all choices of $x_{\mathrm{f}}$.

\section{(b) Computation of optimal state-feedback control}

In the previous section, we showed analytically that the system under consideration is stabilizable for any value of $\mu_{0}$ and all choices of $x_{\mathrm{f}}$. One might think that the issue of characterizing stabilizability for the present system would be closed at this point. However, the present control problem is found to be increasingly delicate to solve numerically as the Reynolds number is increased, to the point that incorrect conclusions about stabilizability can be drawn from numerical calculations unless such calculations are interpreted properly. In the two sections that follow, we thus interpret the results from two different numerical characterizations of the stabilizability property.

We first attempt to compute stabilizing feedback for the discretized CGL equation using optimal control theory. This approach involves the choice of a quadratic cost function $\mathcal{J}$ weighing together a generalized state 'energy' and a measure of the control effort,

$$
\mathcal{J}=\int_{0}^{\infty}\left[\boldsymbol{x}^{*} Q \boldsymbol{x}+\boldsymbol{u}^{*} R \boldsymbol{u}\right] \mathrm{d} t
$$

In the present work, we chose $R=\ell^{2} I$ and $Q=\mathcal{Q}$ (determined in the previous section), so that $\boldsymbol{x}^{*} Q \boldsymbol{x}$ is simply a numerical approximation of $\int_{-\infty}^{\infty}|\psi|^{2} \mathrm{~d} x$. The design parameter $\ell$ allows us to adjust the resulting control magnitude; in the limit $\ell \rightarrow \infty$, the minimal-energy stabilizing control feedback is obtained. Optimal control theory allows us to compute the control forcing $\boldsymbol{u}$ that minimizes the cost function $\mathcal{J}$. This solution is conveniently represented in the state-feedback form $\boldsymbol{u}=K \boldsymbol{x}$, where the gain matrix $K$ is computed using the unique positive-definite solution $X$ to the algebraic Riccati equation (ARE)

$$
A^{*} X+X A-X B R^{-1} B^{*} X+Q=0 \quad \Rightarrow \quad K=-B^{*} R^{-1} X .
$$

Optimal control theory provides an alternative method of determining the stabilizability of the matrix pair $(A, B)$, since, at least in theory (i.e. using infinite-precision arithmetic), a stabilizing positive-definite solution $X$ to the matrix equation given above exists if and only if $A$ is stabilizable by control input through $B$, independent 


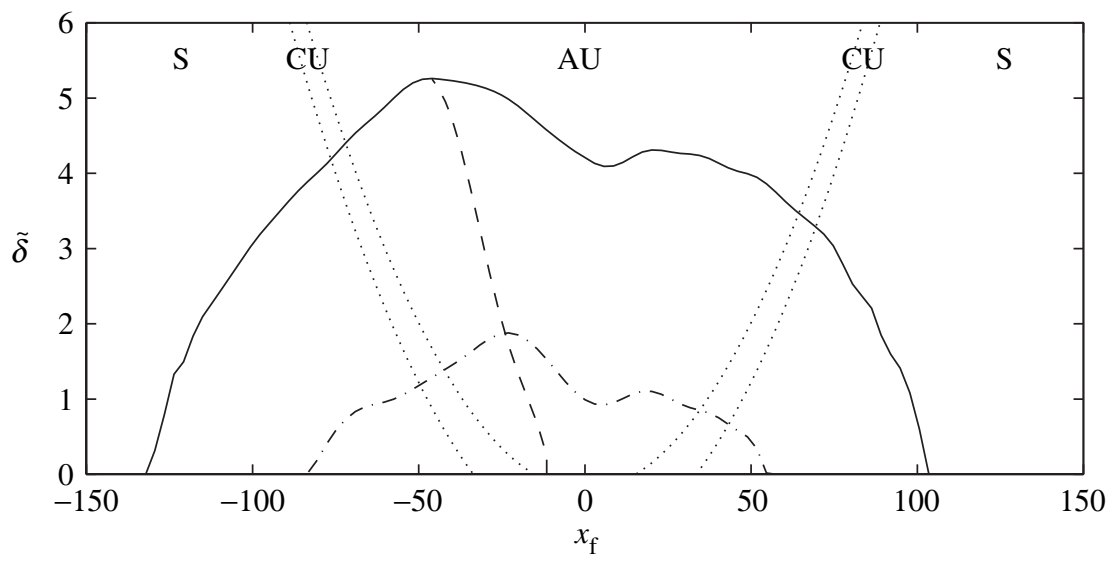

Figure 1. Effective upper bound $\tilde{\delta}\left(x_{\mathrm{f}}\right)$ of supercriticality $\delta$ above which a stabilizing solution $X>0$ to the ARE could not be found using double- (solid line) and single- (dot-dashed line) precision arithmetic. Also indicated (dashed line) is the optimal actuator location (as a function of $\delta$ ) for minimization of $\mathcal{J}$, taking $Q=\mathcal{Q}$ (defined in $\S 3 a$ ); this curve was found to be essentially independent of both $\ell$ and the level of numerical precision used. The boundaries between the different local stability regions are indicated by the dotted lines (S, stable; CU, convectively unstable; AU, absolutely unstable).

of the choice of the positive-definite weighting matrices $R$ and $Q$. Thus, in theory, by determining whether or not a stabilizing positive-definite solution $X$ to (3.4) exists, one may determine the stabilizability of the Ginzburg-Landau operator by pointwise forcing and thus, by iteration, the maximum Reynolds number at which stabilization can be achieved.

The algebraic Riccati equation (3.4) is typically solved using the Schur decomposition technique (Laub 1991). In the present case, this equation was found to become increasingly difficult to solve as the supercriticality is increased. Thus three alternative methods for solving (3.4) were also implemented (see, for example, Jamshidi 1980). As a first alternative, an eigenvalue decomposition technique was used instead of the Schur decomposition. As a second alternative, we chose to transform the continuous-time Riccati equation (3.4) into the corresponding discrete-time Riccati equation, and to solve the latter using a doubling algorithm (Jamshidi 1980). Finally, we implemented a Newton-iterative algorithm to solve (3.4). In all cases, the results obtained agreed within $\pm 6 \%$.

Using double-precision arithmetic, an effective upper bound on the supercriticality was determined, above which a stabilizing solution $X>0$ to the ARE could not be found. This upper bound, which we denote $\tilde{\delta}\left(x_{\mathrm{f}}\right)$, is a function of the forcing position $x_{\mathrm{f}}$, and is displayed as the solid curve in figure 1 . This figure also indicates the boundaries (dotted lines) between the local instability regions and, for each $\delta$, the position of the optimal actuator location (dashed line), which results in the minimum value of the cost function $\mathcal{J}$ over all actuator locations tested.

Somewhat surprising (and, perhaps, somewhat misleading) is the observation that the upper bound $\tilde{\delta}\left(x_{\mathrm{f}}\right)$ indicated in figure 1 , above which a stabilizing solution to the ARE (3.4) could not be found using double-precision arithmetic, appears to be quite independent of several choices in the formulation of the discrete optimal control problem, including the following: 
(i) the positive-definite weighting matrices $Q$ and $R$;

(ii) the number of grid points $N$ used (as long as $N$ is sufficiently large);

(iii) the numerical technique used to perform the spatial discretization; and

(iv) the numerical technique used to solve the ARE.

For each actuator location $x_{\mathrm{f}}$ tested, all calculations of $\tilde{\delta}\left(x_{\mathrm{f}}\right)$ agreed to within $\pm 6 \%$, even as the items listed above were varied. For the parameter values tested, the curve $\tilde{\delta}\left(x_{\mathrm{f}}\right)$ reaches a peak value of $\delta_{\mathrm{m}} \triangleq \max _{x_{\mathrm{f}}} \tilde{\delta}\left(x_{\mathrm{f}}\right)=5.3 \pm 0.3$ with the actuator location $x_{\mathrm{f}}=-47 \pm 1$. For $0<\delta<5.3$, there exists a window of possible actuator locations (indicated by the solid line in figure 1) inside of which stabilizing solutions of the ARE may be found using double-precision arithmetic.

Significantly, the location of the upper bound above which a stabilizing solution to the ARE cannot be found turns out to be a strong function of the level of numerical precision used in the solution of the ARE; a single-precision calculation of this curve is shown as the dashed line in figure 1 . In this case, the curve $\tilde{\delta}\left(x_{\mathrm{f}}\right)$ reaches a peak value of $\delta_{\mathrm{m}}=1.9 \pm 0.1$ with the actuator location $x_{\mathrm{f}}=-23 \pm 1$. Thus, the 'effective upper bound' for stabilizability so determined is not a fundamental stabilization limitation, but rather a persistent artefact of the numerical precision used in the solution of the optimal control problem.

\section{(c) Computation of a minimal-energy stabilizing control feedback rule}

It is a classical result in control theory that, if a minimal-energy stabilizing control feedback rule is used, the eigenvalues of the stabilized closed-loop system $A+B K$ are given by the union of the stable eigenvalues of $A$ and the reflection of the unstable eigenvalues of $A$ into the left half-plane across the imaginary axis. Since we know where the closed-loop eigenvalues of the system are in this case, the feedback gain matrix $K$ may be computed by the process of pole assignment. As shown below, this leads to a simple closed-form expression for the minimal-energy stabilizing control feedback rule.

\section{(i) Review of the solution of the pole-assignment problem}

In order to formulate the pole-assignment problem, we first write down the Hamiltonian that eventually leads to the Riccati equation in the standard derivation of the linear quadratic regulator (LQR) problem,

$$
\left(\begin{array}{cc}
A & -B R^{-1} B^{*} \\
-Q & -A^{*}
\end{array}\right) V=V \Lambda_{\mathrm{cl}}, \quad \text { with } V=\left(\begin{array}{l}
V_{1} \\
V_{2}
\end{array}\right)
$$

where the desired eigenvalues of the closed-loop system, $\lambda_{\mathrm{cl}}$, are listed on the diagonal elements of the diagonal matrix $\Lambda_{\mathrm{cl}}$, and the corresponding eigenvectors of the Hamiltonian are given by the columns of $V$, which is partitioned as indicated. In the pole-assignment problem, we prescribe the closed-loop eigenvalues $\lambda_{\mathrm{cl}}$ in advance, then compute the corresponding eigenvector matrix $V$. As with the formulation of the LQR problem, once this eigenvalue/eigenvector problem is solved, the desired 
feedback rule is given by $\boldsymbol{u}=K \boldsymbol{x}$ with $K=-B^{*} R^{-1} X$, where $X=-V_{2} V_{1}^{-1}$. Multiplying out (3.5), it is easy to show that

$$
\begin{aligned}
A V_{1}-B R^{-1} B^{*} V_{2} & =V_{1} \Lambda_{\mathrm{cl}} \\
-Q V_{1}-A^{*} V_{2} & =V_{2} \Lambda_{\mathrm{cl}}
\end{aligned}
$$

and thus

$$
\begin{aligned}
A Q^{-1}\left(A^{*} V_{2}+V_{2} \Lambda_{\mathrm{cl}}\right)+B R^{-1} B^{*} V_{2} & =Q^{-1}\left(A^{*} V_{2}+V_{2} \Lambda_{\mathrm{cl}}\right) \Lambda_{\mathrm{cl}}, \\
V_{1} & =-Q^{-1}\left(A^{*} V_{2}+V_{2} \Lambda_{\mathrm{cl}}\right) .
\end{aligned}
$$

As $(3.7 a)$ is linear in the unknown matrix $V_{2}$, finding a non-singular solution $V_{2}$ to this equation amounts to a straightforward linear-algebra problem. Once $V_{2}$ is obtained, calculation of $V_{1}$ is trivial using $(3.7 b)$ or, equivalently, $(3.6 a)$. Standard techniques to solve this problem in the general setting discussed here include those by Ackermann (1972) and Kautsky et al. (1985), and are well developed.

\section{(ii) Simplification of the linear-algebra problem in modal form}

In the case in which we are simply trying to compute the minimal-energy stabilizing control feedback, the pole-placement problem reviewed above can be simplified greatly. First note that if

$$
\Lambda=\left(\begin{array}{cccc}
\lambda_{1} & & & 0 \\
& \lambda_{2} & & \\
& & \ddots & \\
0 & & & \lambda_{n}
\end{array}\right) \text { and } V=\left(\begin{array}{cccc}
v_{11} & v_{12} & \cdots & v_{1 n} \\
v_{21} & v_{22} & \cdots & v_{2 n} \\
\vdots & \vdots & \ddots & \vdots \\
v_{n 1} & v_{n 2} & \cdots & v_{n n}
\end{array}\right) \text {, }
$$

then the products $\Lambda V$ and $V \Lambda$ have special structure,

$$
\Lambda V=\left(\begin{array}{cccc}
\lambda_{1} v_{11} & \lambda_{1} v_{12} & \cdots & \lambda_{1} v_{1 n} \\
\lambda_{2} v_{21} & \lambda_{2} v_{22} & \cdots & \lambda_{2} v_{2 n} \\
\vdots & \vdots & \ddots & \vdots \\
\lambda_{n} v_{n 1} & \lambda_{n} v_{n 2} & \cdots & \lambda_{n} v_{n n}
\end{array}\right)
$$

and

$$
V \Lambda=\left(\begin{array}{cccc}
\lambda_{1} v_{11} & \lambda_{2} v_{12} & \cdots & \lambda_{n} v_{1 n} \\
\lambda_{1} v_{21} & \lambda_{2} v_{22} & \cdots & \lambda_{n} v_{2 n} \\
\vdots & \vdots & \ddots & \vdots \\
\lambda_{1} v_{n 1} & \lambda_{2} v_{n 2} & \cdots & \lambda_{n} v_{n n}
\end{array}\right)
$$

We now consider the pole-placement algorithm applied to the equation for the unstable dynamics of the CGL system in modal form, determined analytically in (3.3). Partitioning $V_{2}$ into its respective columns,

$$
V_{2}=\left(\begin{array}{llll}
\xi^{(1)} & \xi^{(2)} & \cdots & \xi^{(n)}
\end{array}\right),
$$

taking $A=\Lambda, B=\bar{B}, Q=I, R=\ell^{2} I$ and $\Lambda_{\mathrm{cl}}=-\Lambda^{*}$, and applying the above relationships, it follows that $(3.7 a)$ may be written as

$$
\left[-\frac{B B^{*}}{\ell^{2}}+\operatorname{diag}\left(d_{1}^{(k)}, d_{2}^{(k)}, \ldots, d_{n}^{(k)}\right)\right] \xi^{(k)} \triangleq M^{(k)} \xi^{(k)}=0,
$$


where

$$
d_{i}^{(k)}= \begin{cases}-\lambda_{i} \lambda_{i}^{*}+\lambda_{i} \lambda_{k}^{*}+\lambda_{k}^{*} \lambda_{k}^{*}-\lambda_{i}^{*} \lambda_{k}^{*} \neq 0 & \text { for } i \neq k, \\ 0 & \text { for } i=k .\end{cases}
$$

Thus the vectors $\xi^{(k)}$ lie in the nullspace of $M^{(k)}$, and may be found by the process of Gaussian elimination, manipulating $M^{(k)}$ to reduced row-echelon form. In the limit $\ell \rightarrow \infty, M^{(k)}$ approaches a diagonal matrix with a zero in the $k$ th diagonal element, and thus $V_{2} \rightarrow I$. In order to avoid taking the difference of two quantities that are almost equal in the computation of $V_{1}$, we return to $(3.6 a)$, which we may now write in the form

$$
\Lambda V_{1}+V_{1} \Lambda^{*}=\frac{B B^{*}}{\ell^{2}} \triangleq \frac{C}{\ell^{2}}
$$

Defining now the $\{i, j\}$ component of $V_{1}$ as $v_{i j}$, it follows immediately that

$$
v_{i j}=\frac{c_{i j}}{\left(\lambda_{i}+\lambda_{j}^{*}\right) \ell^{2}} \triangleq \frac{f_{i j}}{\ell^{2}} .
$$

With $V_{1}=F / \ell^{2}$ and $V_{2}=I$, it follows that $X=-\ell^{2} I F^{-1}$ and thus $\bar{K}=-B X / \ell^{2}=$ $-B^{*} F^{-1} A$.

The resulting closed-form solution for the minimal-energy stabilizing control feedback is now summarized.

Theorem 3.1. Consider a stabilizable system with no pure imaginary open-loop eigenvalues, for which the dynamics of the unstable modes of the open-loop system may be written in the form $\dot{\chi}=\Lambda \chi+\bar{B} \boldsymbol{u}$, where the diagonal matrix $\Lambda$ contains the unstable eigenvalues of the open-loop system, which are assumed to be distinct. Define $C=\bar{B} \bar{B}^{*}$ and compute a matrix $F$ with components $f_{i j}=c_{i j} /\left(\lambda_{i}+\lambda_{j}^{*}\right)$. The minimal-energy stabilizing feedback controller is then given by $\boldsymbol{u}=\bar{K} \chi$, where $\bar{K}=\bar{B}^{*} F^{-1}$.

Proof. The proof follows immediately from the derivation presented above.

In the continuous setting, by the modal decomposition and orthogonality property developed in $\S 3 a, \chi_{k}=\left\langle\eta_{k}, \psi\right\rangle$, and thus the control feedback in the continuous setting is

$$
u=\bar{K}\left(\begin{array}{c}
\left\langle\eta_{1}, \psi\right\rangle \\
\left\langle\eta_{2}, \psi\right\rangle \\
\vdots \\
\left\langle\eta_{n}, \psi\right\rangle
\end{array}\right) .
$$

In the discrete setting, the corresponding modal decomposition and orthogonality property leads to $\chi_{k}=\left(\boldsymbol{s}^{(k)}\right)^{*} \mathcal{Q} \boldsymbol{x}$, and thus the corresponding control feedback rule in the discrete setting is

$$
\boldsymbol{u}=K \boldsymbol{x}, \quad \text { where } K=\bar{K} S^{*} Q, \quad S=\left(\begin{array}{llll}
\boldsymbol{s}^{(1)} & \boldsymbol{s}^{(2)} & \ldots & \boldsymbol{s}^{(n)}
\end{array}\right) .
$$




\section{(iii) Numerical results}

The closed-form solution for $K$ described above was applied to the modal form of the CGL equation, which may be determined analytically (see (3.3)), for a vari-

ety of values of supercriticality $\tilde{\delta}$ and forcing locations $x_{\mathrm{f}}$. Remarkably, numerical calculations of the minimal-energy stabilizing control feedback were found to break down (failing to provide a stable closed-loop system matrix $A+B K$ ) for values of $\tilde{\delta}$ and $x_{\mathrm{f}}$ that accurately coincide with the effective upper bounds for stabilizability obtained by solving the Riccati equation (3.4), as displayed in figure 1, using both single- and double-precision arithmetic. We reach the same conclusion as in the previous section: the stabilizability limit so determined is not a fundamental stabilization limitation, but a persistent artefact of the finite-precision arithmetic used in the numerical computations.

\section{Actuator placement}

It is well known that adjoint eigenfunctions, when properly defined, have a direct relation with the sensitivity of the corresponding system eigenfunctions to applied forcing (see, for example, Park 1994). Chomaz et al. (1990) quantified this property in the case of the CGL equation, showing that the amplitude of the $n$th adjoint eigenfunction at a given position represents, in a quantifiable manner, the magnitude of the response of the $n$th system eigenfunction to small amounts of actuator forcing at this location. Recall that the adjoint eigenfunctions are the system eigenfunctions for the adjoint CGL equation, which may be obtained simply by changing $\{U, \mu, \nu\}$ to $\left\{-U, \mu^{*}, \nu^{*}\right\}$ in (2.1). Because of the change of sign in the advection term, the support of the adjoint eigenfunctions (which is straightforward to calculate using (2.2)) typically lies upstream of the peak of local instability in the CGL system.

We now extend this analysis to the closed-loop setting and to the case in which many system eigenfunctions need to be stabilized. As mentioned previously, the amplitude of each adjoint eigenfunction along the $x$-axis may be interpreted as the sensitivity of the corresponding unforced system eigenfunction to small amounts of control forcing as a function of the forcing location. Thus, in order to stabilize several unstable system eigenfunctions, it is necessary that the actuator lies within the domain of support of each of the corresponding adjoint eigenfunctions. As several of these domains of support overlap (figure 2), it is possible to stabilize several unstable system eigenfunctions simultaneously with a single appropriately situated localized actuator.

Note that, in order to stabilize a particular mildly unstable system eigenfunction, it is not necessary that the actuator be located near the maximum amplitude of the corresponding adjoint eigenfunction; this only denotes where the influence of a small amount of forcing on the system eigenfunction is maximum. Thus, as indicated in figure 1 , in the slightly supercritical case $(0<\delta \ll 1)$, the window of actuator locations that can be used to stabilize the system extends well into the locally stable regions of the system, far upstream and downstream of the maximum of the first adjoint eigenfunction located at $x=-12.8$. In this case, the optimum location for the actuator for minimizing $\mathcal{J}$ (illustrated by the dashed line in figure 1 ) is $x_{\mathrm{f}}=-11.6 \pm 0.7$, which is slightly downstream of the maximum of the first adjoint eigenfunction; a similar result was observed by Chomaz et al. (1990). 


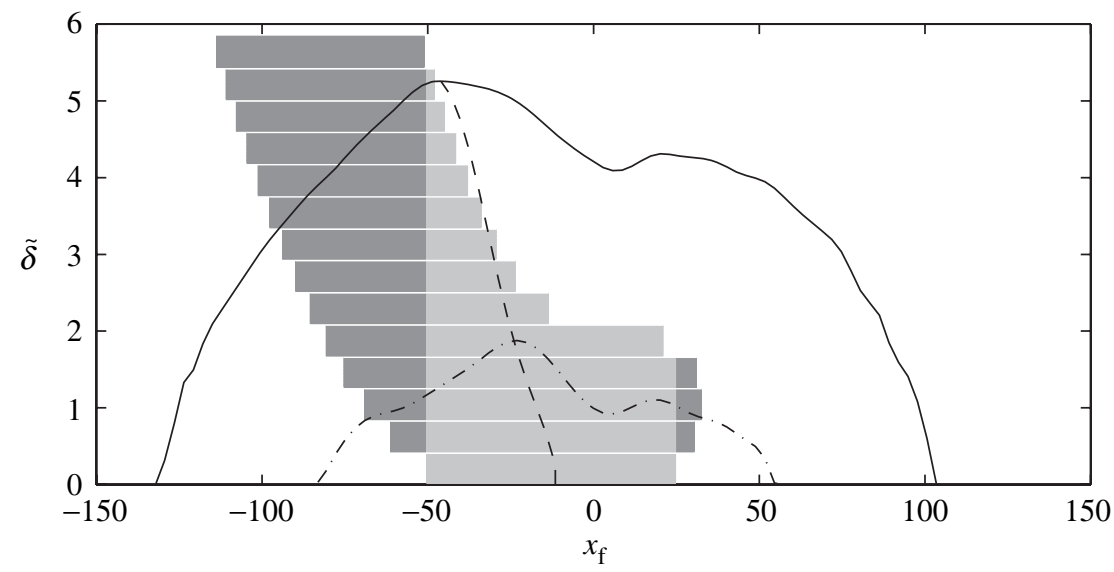

Figure 2. Principal support of the first 14 adjoint eigenfunctions $\eta_{k}$ as a function of $x$. The lower edge of each bar demarcates the supercriticality $\delta$ at which the corresponding system eigenfunction becomes unstable. The horizontal extent of each bar demarcates the region that contains $99 \%$ of the energy of each adjoint eigenfunction centred around its maximum value; each bar includes a portion that has a non-zero intersection with the support of all the adjoint eigenfunctions below it (light grey) and a portion outside of this intersection (dark grey). The stabilization limitations using single- and double-precision arithmetic from figure 1 have been added for interpretation, as well as the optimal actuator location.

Theoretically (i.e. using infinite-precision arithmetic), the adjoint eigenfunctions are never exactly zero on the real axis in the present system. Thus the present system should be stabilizable for any values of $\mu_{0}$ and $x_{\mathrm{f}}$, as discussed in $\S 3 a$. However, as illustrated in (3.2), each adjoint eigenfunction decreases to zero exponentially away from its maximum. Thus the control authority of localized forcing on the unstable eigenfunctions becomes exponentially small far from the maximum of the corresponding adjoint eigenfunctions. As a consequence, using finite-precision arithmetic, effective controls may be computed for only a limited range of values of both $\mu_{0}$ and $x_{\mathrm{f}}$.

Figure 2 indicates the 'principal support' of the first 14 adjoint eigenfunctions, where the 'principal support' $\Omega$ is defined as that region, centred at the maximum value of the adjoint eigenfunction, which contains $99 \%$ of the energy of that adjoint eigenfunction (that is, $\int_{\Omega}\left|\eta_{\kappa}\right|^{2} \mathrm{~d} x=0.99 \int_{-\infty}^{\infty}\left|\eta_{\kappa}\right|^{2} \mathrm{~d} x$ ). It is seen that the first system eigenfunction for which the principal support of the corresponding adjoint eigenfunction does not have an intersection with the principal support of the previous adjoint eigenfunctions is the 14th, and that this eigenmode goes unstable in the uncontrolled CGL system when $\delta=5.42$. This value compares fairly well with the effective stabilizability limit obtained with double-precision arithmetic, $\delta_{\mathrm{m}}=5.3$. The intersection of the supports of the first 13 adjoint modes extends from $x=-50.4$ to $x=-47.8$, which also compares favourably with the optimum forcing location $x_{\mathrm{f}}=-47 \pm 1$.

Figure 3 indicates the principal support of the first 22 adjoint eigenfunctions when the definition of 'principal support' is modified to be that region which contains $99.9 \%$ of the energy of each adjoint eigenfunction. It is seen that, in this case, the first system eigenfunction for which the principal support of the corresponding adjoint eigenfunction does not have an intersection with the principal support of the previous 


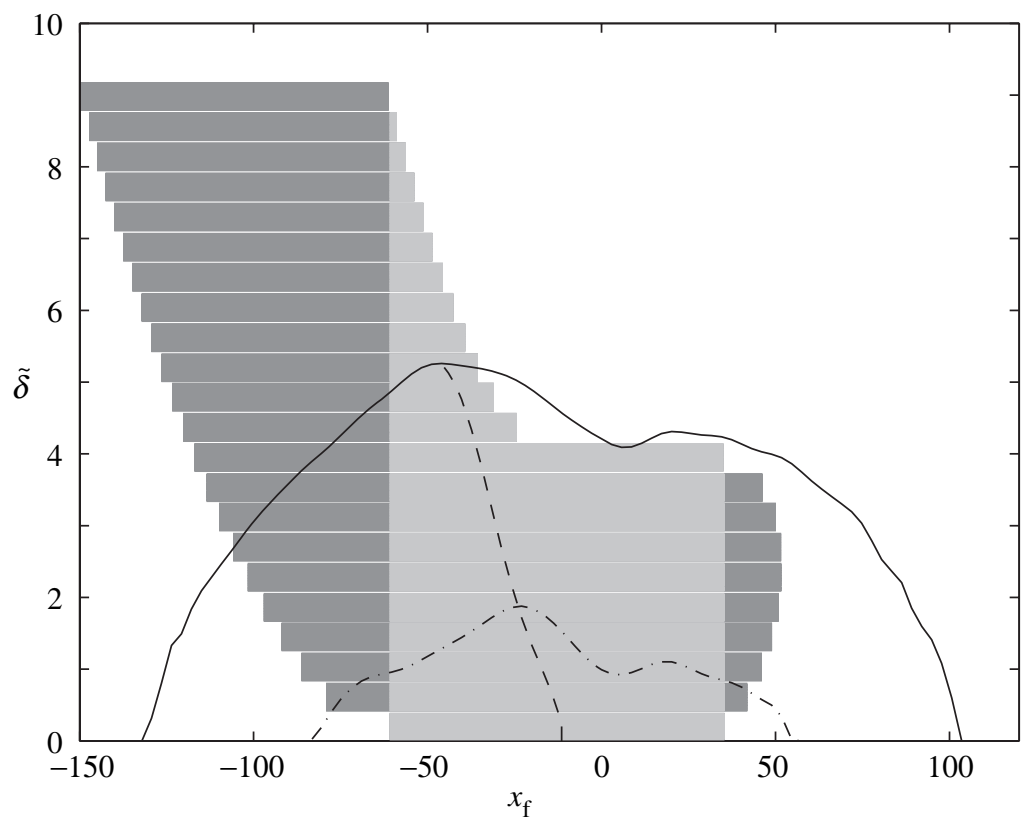

Figure 3. Same as figure 2, with the principal support redefined as the region that contains $99.9 \%$ of the energy of each adjoint eigenfunction.

adjoint eigenfunctions is the 22nd, and that this eigenmode goes unstable in the uncontrolled CGL system when $\delta=9.17$.

We thus see that studying the degree of overlap of the 'principal support' of the adjoint eigenfunctions corresponding to the open-loop unstable system eigenmodes provides valuable insight into the reason for the 'effective stabilizability limit' of the system when controls are computed using finite-precision arithmetic. However, such a characterization in itself does not provide a quantitative prediction of this bound, as it depends on the definition of the 'principal support' of the adjoint eigenfunctions.

\section{Non-normality of the closed-loop system}

The previous section discussed an open-loop characterization of an effective stabilization limitation based on the overlap of the 'principal support' of the adjoint eigenfunctions corresponding to the unstable modes of the system. This is an a priori analysis technique in the sense that it can be performed before any control algorithm is actually computed. An a posteriori observation of the closed-loop (controlled) CGL system can also be used to improve our understanding of this limitation.

As mentioned previously, when an unstable system matrix $A$ is stabilizable by control input through $B$, the minimal-energy stabilizing control feedback (i.e. the optimal control feedback computed in the limit of large $\ell$ ) will leave the stable eigenmodes of the system unchanged, and will reflect the unstable eigenvalues of $A$ across the imaginary axis. Recall from $\S 2$ that the eigenvalues of the open-loop CGL equation may be computed analytically. The symmetric reflection property of the minimal-energy optimal control solution is illustrated in figure 4 , taking $\ell=10^{4}$ and $\delta=3$, for the first 20 eigenvalues of the CGL equation. In this minimal-energy 


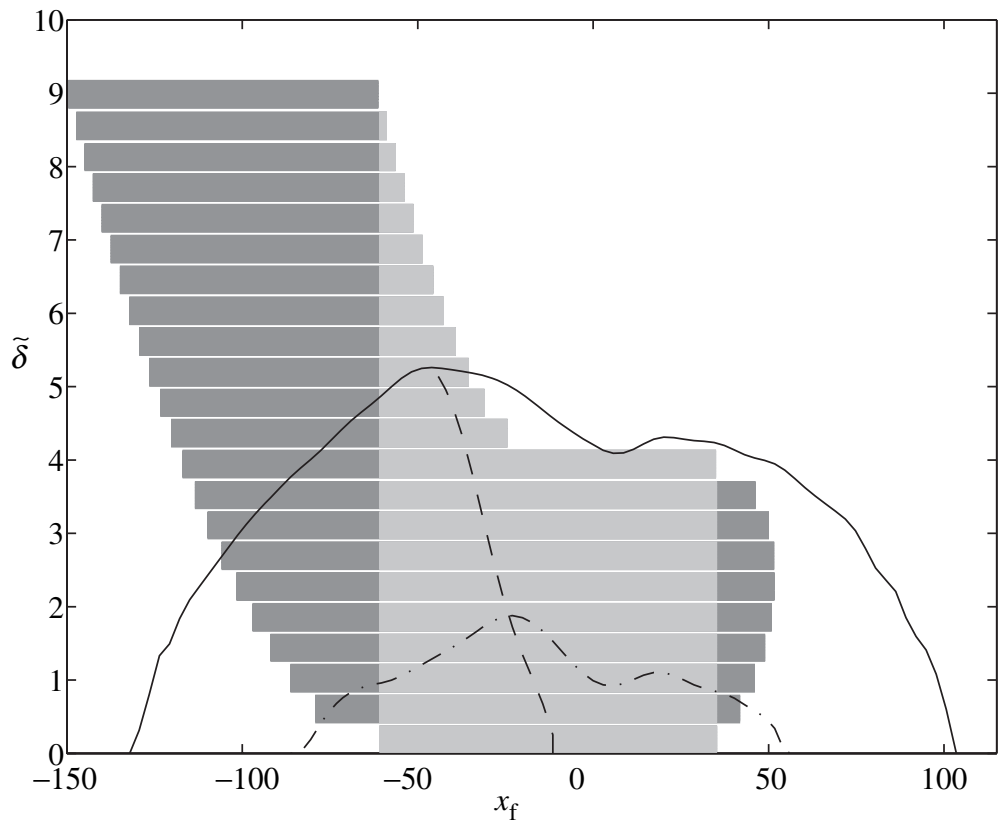

Figure 4. Locus of the first 20 eigenvalues of the CGL operator with supercriticality $\delta=3$ before (pluses) and after (circles) optimal control is applied (with $\ell=10^{4}$ and $x_{\mathrm{f}}=47$ ). Note that, in this minimal-energy optimal control setting, the stable eigenmodes of the system matrix are unchanged, and the unstable eigenvalues of the system matrix are reflected across the imaginary axis.

optimal control setting, it is quite easy to track numerically the effect of the control on each individual system eigenfunction.

Figure 5 displays the amplitude of the two most unstable open-loop eigenfunctions before and after control is applied for several values of $\delta$, again taking $\ell=10^{4}$, with double-precision arithmetic. The discontinuity in the slope of the closed-loop eigenmodes is due to the Dirac delta function introduced in the CGL equation as the forcing. The main point of figure 4 is that, though the amplitudes of the two modes are of quite different shape before application of the control, in the closedloop setting (after application of the control), they tend toward the same shape as $\delta$ increases; this property is also observed for the phase of the two modes. Since the corresponding eigenvalues remain well separated even in closed loop (see figure 4), the closed-loop system becomes what has become known in the fluid-mechanics community as a 'highly non-normal' operator. In this situation, a particular (destructive) linear combination of these two stable eigenmodes as an initial condition can have almost zero initial energy, but can lead to very large transient energy growth, possibly triggering nonlinear instability. Characterizations of both open- and closed-loop system non-normality have recently received a tremendous amount of attention in the fluid-mechanics community (see, for example, Butler \& Farrell 1992; Trefethen et al. 1993; Bewley \& Liu 1998; Bamieh \& Dahleh 2001; Schmid \& Henningson 2001). In the present problem, such a characterization allows interpretation of the stabilization limitation as an approach toward a defective closed-loop system matrix $A+B K$. 


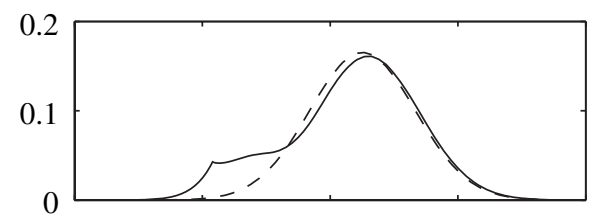

(a)
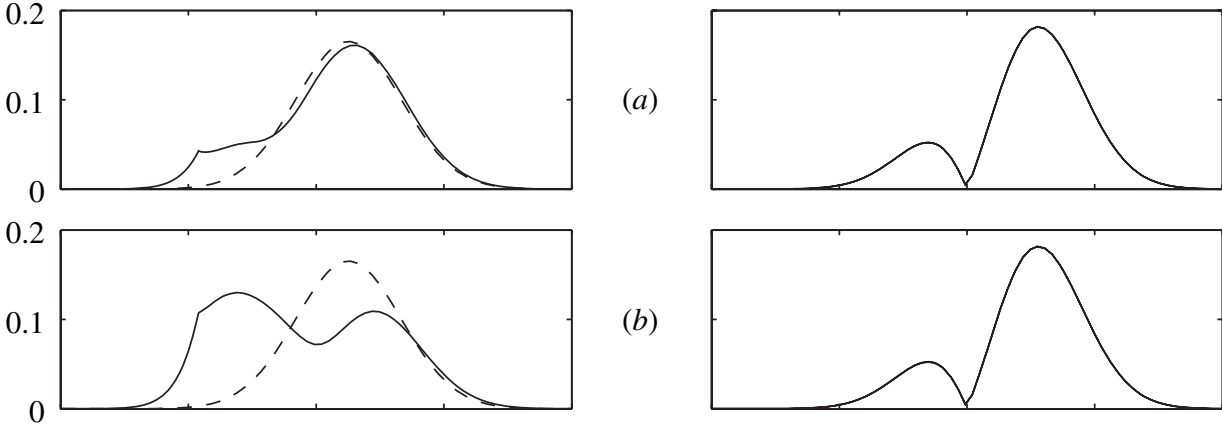

(b)
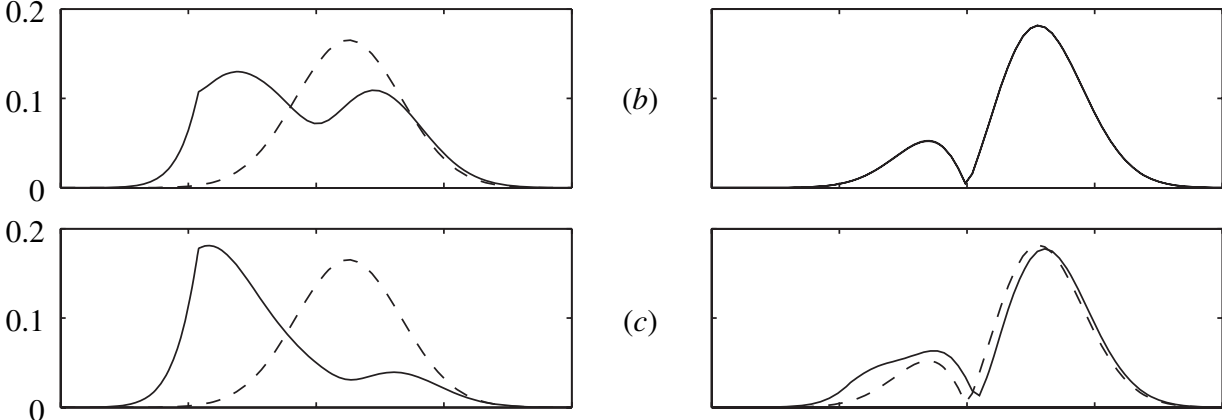

(c)
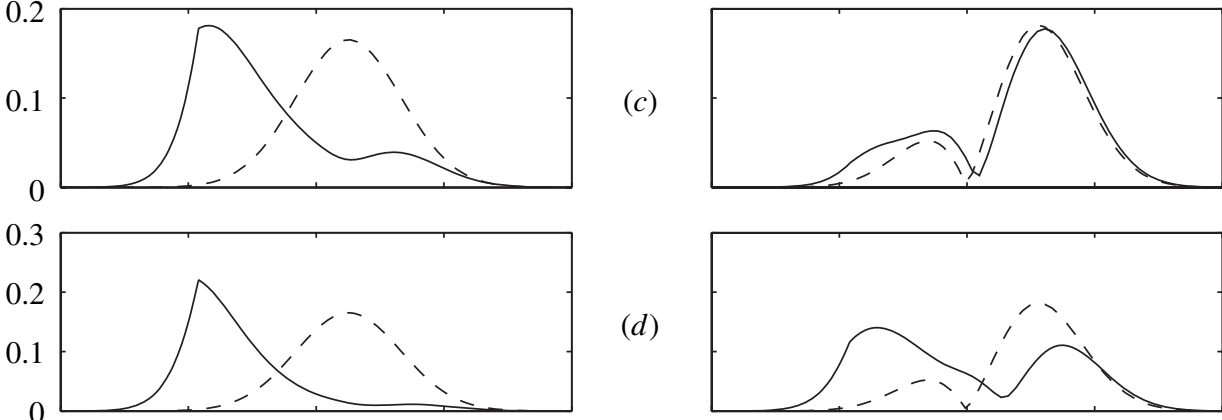

$(d)$
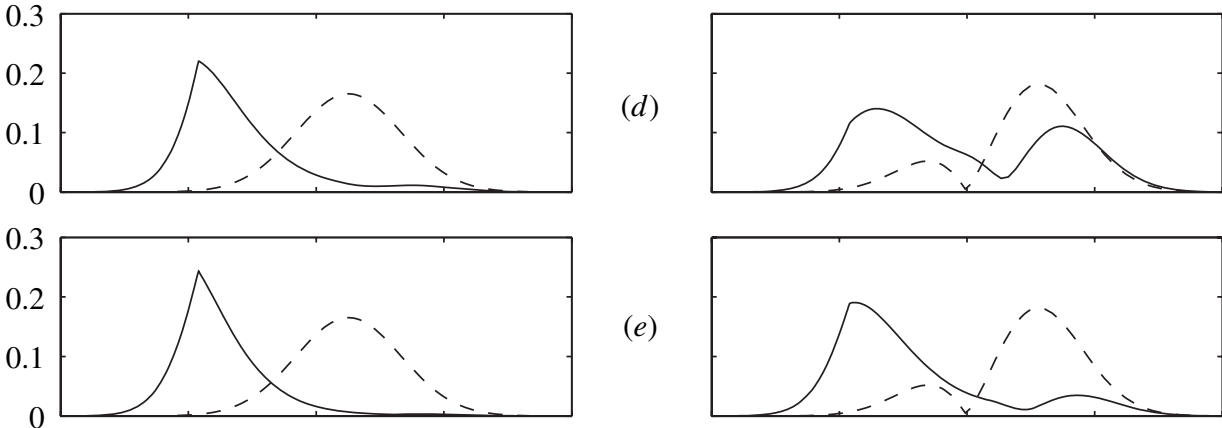

$(e)$
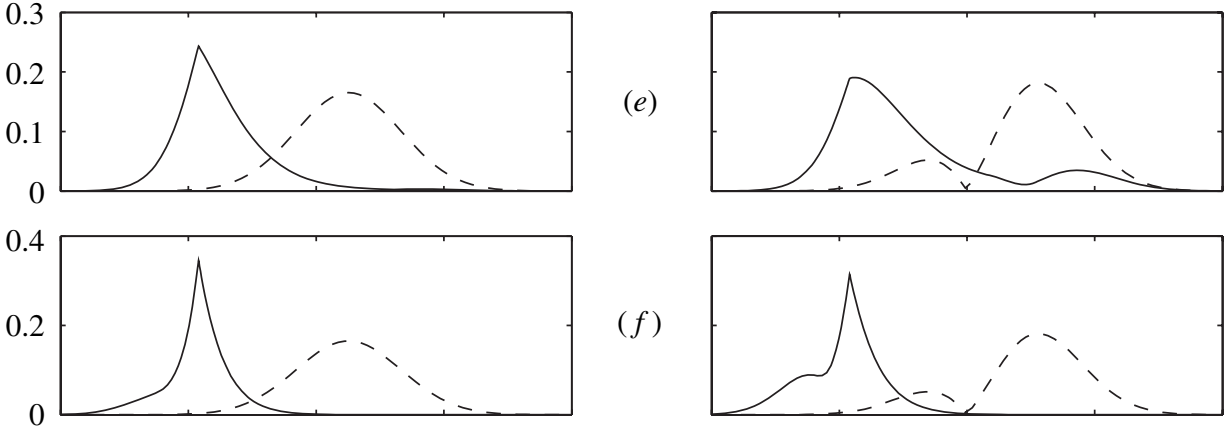

$(f)$
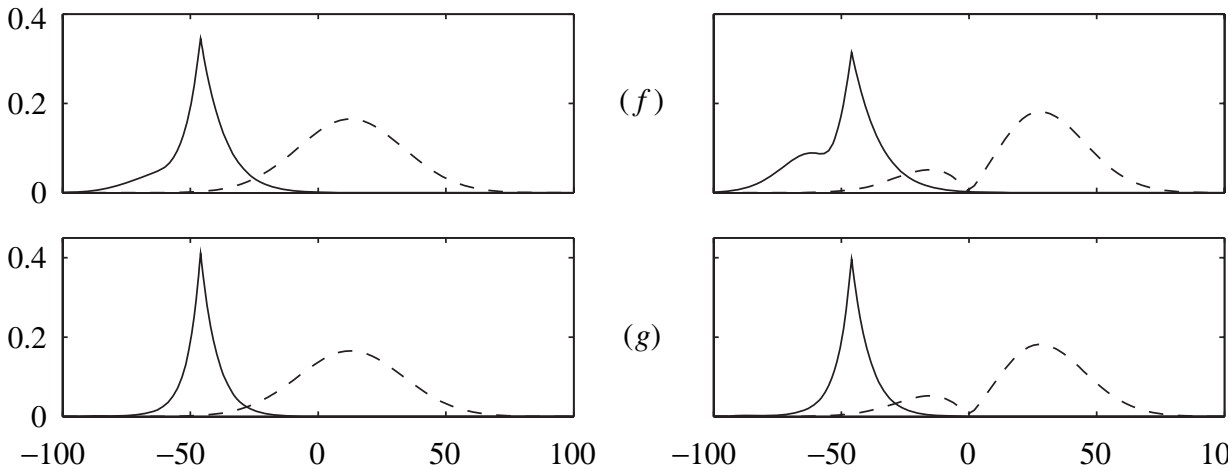

( $g$ )

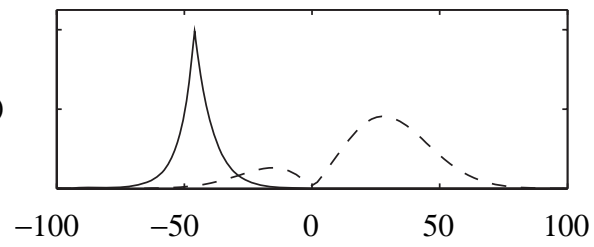

Figure 5. Amplitude of the two most unstable open-loop eigenfunctions before (dashed line) and after (solid line) optimal control is applied for seven values of the supercriticality $\delta$ (with $\ell=10^{4}$, $x_{\mathrm{f}}=-47$ and double-precision arithmetic). Left, most unstable open loop eigenfunction; right, second most unstable. The eigenmodes are scaled to be of unit norm (that is, $\left|\left\langle\tilde{\phi}_{\iota}, \tilde{\phi}_{\iota}\right\rangle\right|=1$ ). Values of $\delta$ are $(a) 0.01,(b) 0.25,(c) 0.5,(d) 0.75,(e) 1,(f) 2.5,(g) 4$.

Table 1 displays the scalar products of the first system eigenfunction with the following four system eigenfunctions (ordered by the real part of their open-loop eigenvalues), before and after control is applied, for various values of the supercriticality $\delta$. These five eigenfunctions become numerically indiscernible with double-precision 
Table 1. Scalar product of the most unstable open-loop eigenfunction $\tilde{\phi}_{0}$ with the four most unstable open-loop eigenfunctions, $\tilde{\phi}_{1}, \tilde{\phi}_{2}, \tilde{\phi}_{3}$ and $\tilde{\phi}_{4}$, after optimal control is applied as a function of the supercriticality $\delta$ (with $\ell=10^{4}, x_{\mathrm{f}}=-47$ and double-precision arithmetic)

(The modes are scaled to be of unit norm.)

\begin{tabular}{ccccc}
\hline$\delta$ & $\left|\left\langle\tilde{\phi}_{0}, \tilde{\phi}_{1}\right\rangle\right|$ & $\left|\left\langle\tilde{\phi}_{0}, \tilde{\phi}_{2}\right\rangle\right|$ & $\left|\left\langle\tilde{\phi}_{0}, \tilde{\phi}_{3}\right\rangle\right|$ & $\left|\left\langle\tilde{\phi}_{0}, \tilde{\phi}_{4}\right\rangle\right|$ \\
\hline 0 & 0.6567 & 0.5028 & 0.3558 & 0.2431 \\
1 & 0.9549 & 0.3292 & 0.1037 & 0.0548 \\
2 & 0.9291 & 0.4964 & 0.3826 & 0.2790 \\
3 & 0.9980 & 0.9804 & 0.8411 & 0.4677 \\
4 & 0.9999 & 0.9973 & 0.9924 & 0.9411 \\
5 & 0.9999 & 0.9999 & 0.9979 & 0.9930 \\
5.1 & 0.9999 & 0.9999 & 0.9992 & 0.9988 \\
5.2 & 0.9999 & 0.9999 & 0.9999 & 0.9999 \\
\hline
\end{tabular}

arithmetic when $\delta=5.20 \pm 0.2$. (The uncertainty accounts for the difficulty of precisely resolving the eigenvectors close to the stabilization limitation.) This result coincides closely with the stabilization limitation, $\delta_{\mathrm{m}}=5.30 \pm 0.3$.

\section{Discussion}

A potentially important role for control theory in fluid mechanics is the prediction and characterization of fundamental performance and stabilization limitations inherent in fluid-mechanical systems. Such limitations are independent of the particular control approach chosen and thus provide a priori estimates as to the possible engineering benefits that might be realized in the search for effective control strategies. This paper represents one of the first attempts to quantify such a fundamental limitation in a spatially distributed model of a fluid-mechanical system. The CGL equation was chosen as a model of inhomogeneous weakly non-parallel flows, and the focus of the article was placed on the fundamental issues pertaining to the decay of stabilizability with Reynolds number. The main results of this paper are as follows.

(i) The chosen system is (theoretically) linearly stabilizable for all values of the bifurcation parameter and the forcing location. However, finite-precision numerical computations of both the optimal control feedback and the minimalenergy stabilizing control feedback display an effective stabilizability limit. The effective stabilizability limit so obtained appears to be only very weakly dependent on the weights chosen in the formulation of the control problem and the numerical methods used for both the discretization of the system and the solution of the result control equations. This apparent limitation, though persistent, is simply an artefact of the numerical precision chosen, and changes significantly when a different numerical precision is used. As a consequence, it does not represent a fundamental feature of the system considered.

(ii) An open-loop analysis based on the overlap of open-loop adjoint eigenfunctions allows approximate identification of the optimal actuator placement for minimization of the cost function and a characterization of a fundamental closedloop property, that is, the limiting Reynolds number beyond which stabilizability of the system breaks down. This characterization is a function of the 
definition the 'principal support' of the adjoint eigenfunctions, and thus does not reveal a quantitative prediction of this effective limitation, which itself is a function of numerical precision used. Though not presented here, further numerical calculations show that the optimal actuator placement and characterization of the effective stabilization limitation in the case of two actuators follows in a similar fashion.

(iii) An examination of the eigenmodes of the closed-loop system reveal that the decay of stabilizability in the system considered as the bifurcation parameter is increased is characterized by heightened non-normality of the closed-loop eigenmodes and an approach toward a defective closed-loop system matrix $A+B K$. As the effective stabilization limitation is approached, the shapes of the leading closed-loop system eigenfunctions become numerically indiscernible.

The linear CGL equation studied in this paper approximately models small perturbations of spatially developing flow systems over only a limited range of Reynolds numbers. The conclusions about high-Reynolds-number behaviour drawn from the present study of the linear CGL equation are thus not directly applicable to real flow systems. However, the linear-analysis approaches developed in this paper extend naturally to the study of both stabilization and detection (that is, the stabilization of estimation error) in a range of spatially developing flow models, including linearizations of the full three-dimensional Navier-Stokes equation itself. In such systems, the system eigenfunctions and adjoint eigenfunctions are generally not available analytically. However, the leading eigenfunctions and adjoint eigenfunctions (that is, those corresponding to the open-loop unstable eigenvalues) may be computed numerically using the implicitly restarted Arnoldi method applied to standard computational fluid dynamics codes which accurately model the flow and adjoint systems of interest. Extension of the analysis approaches developed in this paper to more physically relevant numerical models of spatially developing flow systems is thus straightforward; a few such extensions are currently well underway, and will be reported in a forthcoming paper. The three main results of the present study, as itemized above, are also expected when the same analysis approaches are applied to higher-fidelity models of spatially developing flow systems which exhibit similar dynamics, such as jets and wakes. Specifically, in such systems, it is anticipated that the following hold.

(i) Linear stabilizability/detectability is lost gradually as the Reynolds number is increased. That is, there is no critical Reynolds number above which such systems fundamentally become linearly unstabilizable/undetectable. However, practically speaking, linear stabilization/detection of such systems becomes impossible at sufficiently high Reynolds number.

(ii) Analysis of the overlap of the open-loop adjoint eigenfunctions corresponding to the open-loop unstable eigenvalues reveals effective actuator locations for the linear stabilization of the system.

(iii) The linear closed-loop system becomes increasingly non-normal as the Reynolds number is increased. The approach towards loss of stabilizability (or detectability) is accompanied by an approach towards a defective closed-loop system matrix $A+B K$ (or $A+L C$ ) regardless of the control design technique used. 
The identification of these inherent properties in high-fidelity models of spatially developing flow systems should provide us with important new insights into the design of effective control strategies.

The authors gratefully acknowledge many fruitful discussions with Jean-Marc Chomaz, Carlo Cossu, François Gallaire, Patrick Huerre and Benoit Pier regarding the CGL equation, with Roberts Bitmead and Skelton regarding linear control theory, and with Patricia Catalifaud regarding the Fortran implementation. Generous funding from both AFOSR (Belinda King) and CTE, Ecole des Mines de Paris, is also gratefully acknowledged.

\section{References}

Ackermann, J. 1972 Der Entwurf Linearer Regulungssysteme in Zustandsraum. Regelungstechnik Prozessdatenverarbeitung 7, 297-300.

Bamieh, B. \& Dahleh, M. 2001 Energy amplification in channel flows with stochastic excitation. Phys. Fluids 13, 3258-3269.

Bewley, T. R. \& Liu, S. 1998 Optimal and robust control and estimation of linear paths to transition. J. Fluid Mech. 365, 305-349.

Butler, K. M. \& Farrell, B. F. 1992 Three-dimensional optimal perturbations in viscous shear flows. Phys. Fluids A 4, 1637-1650.

Chomaz, J. M., Huerre, P. \& Redekopp, L. G. 1987 Models of hydrodynamics resonances in separated shear flows. In Proc. 6th Symp. on Turbulent Shear Flows, Paul Sabatier University, Toulouse, France, pp. 321-326.

Chomaz, J. M., Huerre, P. \& Redekopp, L. G. 1990 The effect of nonlinearity and forcing on global modes. In New trends in nonlinear dynamics and pattern-forming phenomena (ed. P. Coullet \& P. Huerre), pp. 259-274. New York: Plenum.

Chomaz, J. M., Huerre, P. \& Redekopp, L. G. 1991 A frequency selection criterion in spatially developing flows. Stud. Appl. Math. 84, 119-144.

Hammond, D. A. \& Redekopp, L. G. 1997 Global dynamics of symmetric and asymmetric wakes. J. Fluid Mech. 331, 231-260.

Huerre, P. \& Monkewitz, P. A. 1985 Absolute and convective instabilities in free shear layers. J. Fluid Mech. 159, 151-168.

Huerre, P. \& Monkewitz, P. A. 1990 Local and global instabilities in spatially developing flows. A. Rev. Fluid Mech. 22, 473-537.

Jamshidi, M. 1980 An overview on the solutions of the algebraic matrix Riccati equation and related problems. In Large scale systems, vol. 1, pp. 167-192.

Kautsky, J., Nichols, N. K. \& Van Dooren, P. 1985 Robust pole assignment in linear state feedback. Int. J. Control 41, 1129-1155.

Laub, A. J. 1991 Invariant subspace methods for the numerical solution of Riccati equations. In The Riccati equation (ed. S. Bittaini, A. J. Laub \& J. C. Willems), pp. 163-196. Springer.

Min, C. \& Choi, H. 1999 Suboptimal feedback control of vortex shedding at low Reynolds numbers. J. Fluid Mech. 401, 123-156.

Monkewitz, P. A. 1988 The absolute and convective nature of instability in two-dimensional wakes at low Reynolds number. Phys. Fluids 31, 999-1006.

Monkewitz, P. A. 1989 Feedback control of global oscillations in fluid systems. AIAA Paper 890991.

Monkewitz, P. A. 1993 Wake control. In Bluff-body wakes, dynamics and instabilities (ed. H. Eckelmann, J. M. R. Graham, P. Huerre \& P. A. Monkewitz), pp. 227-290. Springer.

Monkewitz, P. A. \& Sohn, K. D. 1988 Absolute instability in hot jets. AIAA J. 26, 911-916.

Monkewitz, P. A., Berger, E. \& Schumm, M. 1991 The nonlinear stability of spatially inhomogeneous shear flows, including the effect of feedback. Eur. J. Mech. B 10, 295-300. 
Monkewitz, P. A., Huerre, P. \& Chomaz, J. M. 1993 Global linear stability analysis of weakly non-parallel shear flows. J. Fluid Mech. 251, 1-20.

Park, D. S. 1994 Theoretical analysis of feedback control of von-Karman vortex street at slightly supercritical Reynolds number. Eur. J. Mech. B 13, 387-399.

Park, D. S., Ladd, D. M. \& Hendricks, E. W. 1994 Feedback control of von Kárman vortex shedding behind a circular cylinder at low Reynolds numbers. Phys. Fluids 6, 2390-2405.

Pier, B. \& Huerre, P. 2001 Nonlinear self-sustained structures and fronts in spatially developing wake flows. J. Fluid Mech. 435, 145-174.

Pier, B., Huerre, P., Chomaz, J. M. \& Couairon, A. 1998 Steep nonlinear gobal modes in spatially developing media. Phys. Fluids 10, 2433-2435.

Roussopoulos, K. 1993 Feedback control of vortex shedding at low Reynolds numbers. J. Fluid Mech. 248, 267-296.

Roussopoulos, K. \& Monkewitz, P. A. 1996 Nonlinear modelling of vortex shedding control in cylinder wakes. Physica D 97, 264-273.

Schmid, P. J. \& Henningson, D. S. 2001 Stability and transition in shear flows. Springer.

Trefethen, L. N., Trefethen, A. E., Reddy, S. \& Driscoll, T. A. 1993 Hydrodynamic stability without eigenvalues. Science 261, 578-584. 


\title{
ERRATUM
}

\section{The decay of stabilizability with Reynolds number in a linear model of spatially developing flows}

\author{
By ERIC Lauga and Thomas R. Bewley
}

Proc. R. Soc. Lond. A 459, 2077-2095 (2003)

Figure 4 is reproduced in its correct form here.

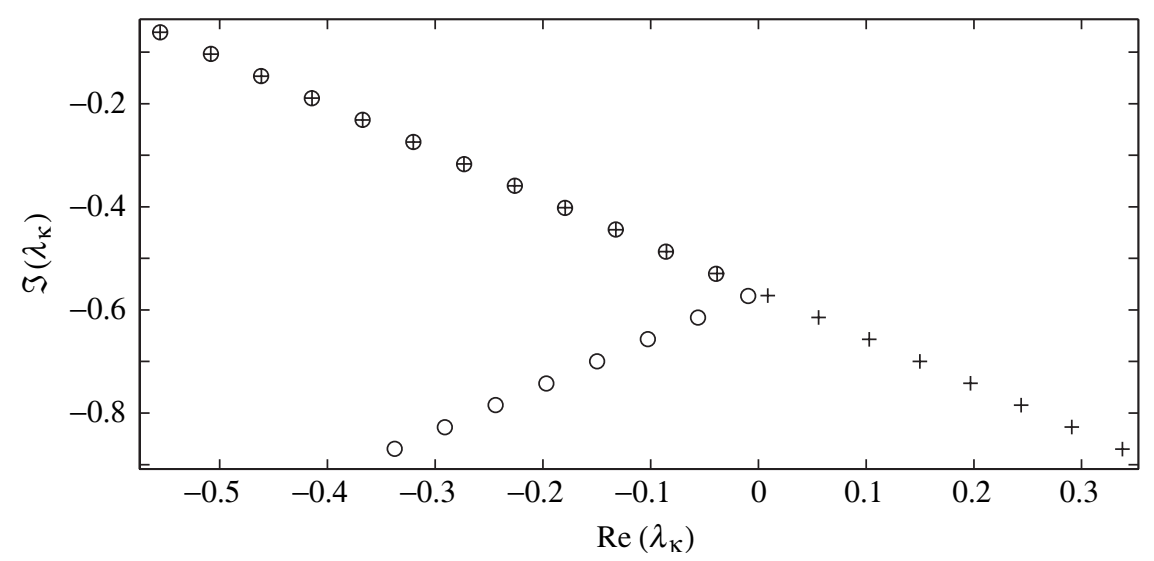

Figure 4. Locus of the first 20 eigenvalues of the CGL operator with supercriticality $\delta=3$ before (pluses) and after (circles) optimal control is applied (with $\ell=10^{4}$ and $x_{\mathrm{f}}=47$ ). Note that, in this minimal-energy optimal control setting, the stable eigenmodes of the system matrix are unchanged, and the unstable eigenvalues of the system matrix are reflected across the imaginary axis. 